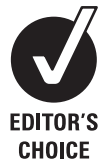

${ }^{1}$ Division of Clinical Neurosciences, University of Edinburgh, Western General Hospital, Edinburgh, UK ;

${ }^{2}$ Academic Medical Centre, University of Amsterdam, Amsterdam, The Netherlands

Correspondence to: Dr F N Doubal, Division of Clinical Neurosciences, University of Edinburgh, Bramwell Dott Building, Western General Hospital, Edinburgh EH4 2XU, UK ;

fergus.doubal@ed.ac.uk

Received 8 May 2008 Revised 16 September 2008 Accepted 22 September 2008 Published Online First

17 October 2008

\title{
Retinal microvascular abnormalities and stroke: a systematic review
}

\author{
F N Doubal, ${ }^{1}$ P E Hokke, ${ }^{2}$ J M Wardlaw ${ }^{1}$
}

\begin{abstract}
Background: Lacunar strokes account for $25 \%$ of ischaemic strokes, but their precise aetiology is unknown. Similarities between the retinal and cerebral small vessels mean that clarification of the exact relationship between retinal microvascular abnormalities and stroke, and particularly with stroke subtypes, may aid understanding of the aetiology of lacunar stroke and stroke risk.
\end{abstract}

Methods: A systematic review of the literature was performed by searching Medline and Embase to October 2007 for studies in humans that investigated the association between retinal microvascular abnormalities and prevalent or incident stroke. Data and calculated summary risk ratios (sRR) were extracted for associations between retinal microvascular abnormalities and stroke, including stroke subtypes where possible, adjusted for key variables. 37 papers from 22 different studies were included with 62975 subjects (mean age 62 years) among whom there were 2893 strokes. Stroke identification and diagnosis methods varied.

Results: Retinopathy was associated with incident stroke (sRR 2.1, 95\% Cl 1.7 to 2.6) and prevalent stroke (sRR $2.5,95 \% \mathrm{Cl} 1.4$ to 4.3 ). Incident stroke was also associated with retinal artery embolism (sRR $2.9,95 \% \mathrm{Cl}$ 1.6 to 5.1 ) and venular widening (sRR $1.4,95 \% \mathrm{Cl} 1.1$ to 1.7). There was significant heterogeneity between studies for some associations. There were no data on retinal microvascular abnormalities and haemorrhagic versus ischaemic stroke or ischaemic stroke subtypes.

Conclusions: Retinal microvascular abnormalities are associated with stroke, but more data are required to clarify associations between specific types of retinal microvascular abnormality and stroke, as well as between different stroke subtypes. Future retinal-stroke studies should concentrate on carefully diagnosing and accurately sub-typing ischaemic stroke.

Lacunar ischaemic stroke accounts for $25 \%$ of all ischaemic strokes, ${ }^{1}$ but its precise aetiology is unknown. ${ }^{2}$ Possible causes include atherothromboembolism, ${ }^{3}$ intracranial large artery stenosis, intrinsic microvascular atheroma, or endothelial dysfunction manifesting either through ischaemia or failure of the blood-brain barrier. ${ }^{4}$

The cerebral blood vessels that, when diseased, give rise to lacunar stroke share many characteristics with retinal vessels, ${ }^{5}$ being developmentally related during embryogenesis and of similar diameter $(50-250 \mu \mathrm{m})$. Retinal vessels are visibly affected by known risk factors for stroke such as diabetes and hypertension. ${ }^{6}$ Therefore, determining any association between retinal microvascular abnormalities and stroke may improve understanding of the nature of the cerebral small vessel changes underlying lacunar ischaemic stroke and aid cerebrovascular and cardiovascular risk profiling.

Several studies have examined a possible link between stroke and the retina, including large epidemiological studies in community-dwelling populations and small detailed studies of patients with specific diseases attending tertiary hospitals. The presence and strength of any reported associations between retinal vascular abnormalities and stroke vary considerably, possibly reflecting the differing populations, methodologies, definitions of disease and abnormalities sought.

Therefore, we performed a systematic review to clarify associations between a range of retinal microvascular abnormalities and any stroke and with specific subtypes of both stroke and ischaemic stroke. We also determined where more information was needed from new studies.

\section{METHODS}

\section{Search strategy}

We searched Ovid Medline (from 1 January 1950) and Embase (from 1 January 1980) to 5 October 2007 for papers investigating associations between retinal microvascular abnormalities and stroke (defined as stroke/transient ischaemic attack/ asymptomatic brain infarct identified on imaging). The electronic search was supplemented by the authors' personal files, hand searching the bibliographies of papers selected from the electronic search, checking references of review articles and by surveillance of key journals from April 2005 to October 2007 (Neurology, Lancet, Brain, Ophthalmology, Stroke, British Journal of Ophthalmology, BMJ, Retina). Two reviewers (FD and $\mathrm{PH}$ ) independently conducted the searches, paper selection and data extraction. A third reviewer (JW) assessed a sample of papers for inclusion, extracted data and adjudicated in any disagreements.

\section{Inclusion criteria}

We included studies published in full which assessed the association between retinal microvascular abnormalities and stroke in adult humans.

\section{Exclusion criteria}

We excluded studies published only as abstracts or presented in conferences without full subsequent publication, review papers, papers dealing with specific single gene-related disorders such as CADASIL, papers studying only macular degeneration and duplicate publications. We excluded papers dealing with retinal microvascular abnormalities and only cerebral white matter disease (leucoaraosis) seen on imaging. 


\section{Definitions}

Stroke is defined as a focal sudden neurological deficit of presumed vascular aetiology with brain imaging excluding other causes.' We defined "stroke" as either a clinically diagnosed stroke or transient ischaemic attack (with or without cerebral imaging) or a cerebral infarct identified on brain imaging without definite associated clinical features being documented. We defined "incident stroke" as strokes which occurred after the patient/subject had been enrolled in the study and "prevalent stroke" as strokes which had preceded patient enrolment in the study.

"Retinal microvascular abnormalities" included retinopathy (microaneurysms, soft and hard exudates, haemorrhages), retinal arteriolar and venular width measurements (arteriovenous ratios (AVR), focal arteriolar narrowing (FAN), arteriovenous nicking $(A V N)$ ), retinal artery $(R A O)$ or vein occlusion (RVO), retinal artery emboli and alterations in retinal vascular geometry (angles or vessel tortuosity).

\section{Paper assessment}

We collected data on a form designed and piloted specifically for this review. We recorded study population details (age, selection criteria, co-morbidities), study design and assessed definitions of stroke and retinal outcomes.

We assigned the reference standard for retinal assessment as high-quality retinal photographs of both eyes (preferably multifield) and blinded assessment of the retinal appearance with a standardised and externally validated tool. We collected data on which retinal features were measured, methods of retinal assessment, use of mydriatics, number of fields imaged per eye and which eyes were photographed, type of camera and field of view.

The reference standard for stroke diagnosis was assessment of the patient by a stroke specialist at the time of the stroke with appropriate cerebral imaging to differentiate haemorrhagic from ischaemic stroke and stroke mimic and, if ischaemic, to identify whether lacunar or large artery ischaemic stroke. We considered the following methods of stroke diagnosis to be less reliable: patient questionnaires, retrospective case note analysis, death certificates and review of centrally held health statistics. We assessed studies for the method of subject selection, the presence of a suitable control group, subtyping of stroke and blinding of assessment of images/patients. We extracted data on numbers with stroke or transient ischaemic attack (TIA) and different retinal features in as much detail as possible, any calculated odds ratios, relative risks or hazards ratios and whether these were unadjusted or adjusted for shared risk factors such as hypertension or diabetes. When counting total numbers of patients and stroke events that contributed to each analysis, we were careful to count patients from studies contributing more than one paper only once to avoid unnecessary bias. We did not have the resources to contact authors of studies to ask regarding missing data or individual patient data.

\section{Statistical analysis}

We summarised the numbers of subjects in the different population samples, with or without various retinal abnormalities and stroke. Stroke outcomes were rare (incidence less than $10 \%$ ), and odds ratios, hazards ratios and relative risk are considered to be equivalent. We converted all ratios to summary risk ratios (sRR) and prepared Forrest Plots using Review Manager Software Version 5 (Cochrane, Oxford) and Excel (version 2003; Microsoft, Redmond, Washington). We used a random effects generic inverse variance method to plot sRR and $95 \%$ CIs using a logarithmic scale from the adjusted ratios and $95 \%$ CI. We used adjusted ratios in preference to unadjusted to best account for differences in risk factors between populations. We tested for associations between stroke and predefined retinal microvascular abnormalities, and then separately examined associations in important predefined subgroups such as incident or prevalent stroke, ischaemic versus haemorrhagic stroke and ischaemic stroke subtypes. We tested for heterogeneity between study results with the $\chi^{2}$ test for heterogeneity with an alpha level for significance set at $p=0.05$. When heterogeneity was detected, we investigated the methodology and definitions of variables used in the heterogeneous studies to identify possible causes.

\section{RESULTS}

\section{Included studies}

The initial search produced 4461 titles and abstracts, subsequent screening of which yielded 52 potentially relevant papers describing probable retinal microvascular abnormalities and stroke for which we assessed the full texts. Of the 52, 15 were excluded (13 were not relevant, and two were in Japanese and not easily translated), leaving 37 papers which met the inclusion criteria. Table 1 documents the main findings of the included papers.

\section{Description of included studies}

Of the 37 papers, 21 originated from large population-based cohort studies (table 2, fig 1). ${ }^{8-28}$ Of the remaining 16 papers, 14 originated from hospital-based studies, ${ }^{29-42}$ one from within a trial of blood pressure reduction ${ }^{43}$ and one from a World Health Organization (WHO) study of follow-up in diabetics. ${ }^{44}$ The papers were from the US, UK, Australia, Japan, Korea, The Netherlands, Saudi Arabia, Greece, Switzerland, Poland, Germany, Croatia, Hong Kong and Cuba.

The 37 papers include 62975 subjects. The mean age of the patients in the $31 / 37$ papers which provided this information was 62.5 years (SD 7.7). Among the 62975 subjects, 1900 had a clinically evident stroke (total of incident and prevalent stroke), and 993 had infarction seen on brain imaging with no stated clinical correlate. We were unable to extract the number of strokes from $6 / 37$ papers. ${ }^{19} 2325363839$ The number of strokes per paper varied from eight to $496 .{ }^{10} 32$

\section{Ascertainment of stroke}

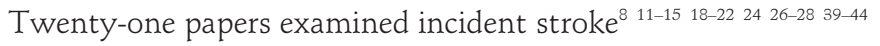
(total population 34 894; 1442 (4.1\%) strokes during follow-up). Fifteen papers examined prevalent stroke 16172325 29-38 (total population 21 950; 501 (2.2\%) had prior stroke), and one paper examined both prevalent and incident stroke. ${ }^{10}$

The definition of stroke varied between the papers. Thirty of the 37 papers defined "stroke" in the index paper or related publications. $^{8-16} 18-24$ 26-30 32-40 Nine of the 37 papers used asymptomatic infarcts seen on brain imaging. ${ }^{9} 102729-31$ 36-38 The other 28 papers assessed some measure of clinical stroke of which only 9/28 papers subtyped stroke into haemorrhagic or ischaemic. $^{814} 15232632-3440$ Of these nine papers, six further subtyped ischaemic strokes, ${ }^{814} 15233440$ but only one paper reported retinal microvascular abnormality differences between the ischaemic stroke subgroups. ${ }^{34}$

The methods of stroke identification varied widely. Two papers used specialist examination at the time of stroke; ${ }^{34} 40$ 
Table 1 Descriptions of included studies arranged by stroke outcome

\begin{tabular}{|c|c|c|c|c|c|c|}
\hline Study & Origin & Size (n) & Retinal feature & Stroke outcome & Association found? & Stroke (n) \\
\hline \multicolumn{7}{|l|}{ Prevalent stroke } \\
\hline Abu El-Asrar et al. ${ }^{32}$ & & 648 & Retinopathy & Prevalent ischaemic stroke & Yes & 8 \\
\hline Petitti and Bhatt ${ }^{33}$ & & 2124 & Retinopathy & Prevalent ischaemic stroke & Yes & 56 \\
\hline Luijckx et $a^{\beta 4}$ & & 59 & Arteriosclerosis & Prevalent ischaemic stroke & No & 59 \\
\hline Hayreh et $a^{35}$ & & 1090 & RVO & Prevalent stroke & Yes & 43 \\
\hline Mitchell et $a l^{25}$ & BMES & 3654 & RVo & Prevalent stroke & Yes & $?$ \\
\hline \multirow[t]{3}{*}{ Klein $e t a^{23}$} & ARIC & 8772 & AVR & Prevalent stroke & No & $?$ \\
\hline & & & AVN & Prevalent stroke & No & \\
\hline & & & FAN & Prevalent stroke & No & \\
\hline Wong et $a l^{17}$ & Ausdiab & 1027 & Retinopathy & Prevalent stroke & Yes & 42 \\
\hline Wong et $a l^{16}$ & CHS & 2050 & Retinopathy & Prevalent stroke & Yes & 133 \\
\hline Kwon et al. ${ }^{29}$ & & 550 & Retinopathy & Prevalent MRI infarct & Yes & 61 \\
\hline Inoue et $a l^{30}$ & & 361 & Retinopathy & Prevalent MRI infarct & Yes & 101 \\
\hline Kobayashi et $a l^{37}$ & & 933 & Arteriosclerosis & Prevalent MRI infarct & Yes & 99 \\
\hline \multirow[t]{4}{*}{ Longstreth et a $\left.\right|^{10}$} & CHS & 1717 & AVR & Prevalent MRI infarct & Yes & 496 \\
\hline & & & AVN & Prevalent MRI infarct & Yes & \\
\hline & & & FAN & Prevalent MRI infarct & No & \\
\hline & & & Retinopathy & Prevalent MRI infarct & No & \\
\hline \multirow[t]{6}{*}{ Cooper et al ${ }^{\beta}$} & ARIC & 1684 & AVR & Prevalent MRI infarct & Yes & 183 \\
\hline & & & FAN & Prevalent MRI infarct & Yes & \\
\hline & & & Haemorrhage & Prevalent MRI infarct & Yes & \\
\hline & & & Soft exudates & Prevalent MRI infarct & No & \\
\hline & & & Microaneurysms & Prevalent MRI infarct & Yes & \\
\hline & & & AVN & Prevalent MRI infarct & Yes & \\
\hline Ueda et $a l^{36}$ & & 185 & RVO/RAO & Prevalent MRI infarct & Not clear & $?$ \\
\hline \multirow[t]{2}{*}{ Kwa et $a f^{38}$} & & 179 & Arteriosclerosis & Prevalent MRI infarct & No & $?$ \\
\hline & & & Art tortuosity & Prevalent MRI infarct & No & \\
\hline Ikeda $e t a^{\beta 1}$ & & 318 & Retinopathy & Prevalent computed tomography infarcts & Yes & 56 \\
\hline \multicolumn{7}{|l|}{ Incident stroke } \\
\hline Cheung et $a l^{\beta}$ & ARIC & 1617 & Retinopathy & Incident ischaemic stroke & Yes & 75 \\
\hline Bruno et al ${ }^{40}$ & & 140 & Retinal emboli & Incident ischaemic stroke & Yes & 19 \\
\hline \multirow[t]{4}{*}{ Wong et $a l^{14}$} & ARIC & 10358 & Retinopathy & Incident ischaemic stroke & Yes & 113 \\
\hline & & & AVN & Incident ischaemic stroke & Yes & \\
\hline & & & FAN & Incident ischaemic stroke & No & \\
\hline & & & AVR & Incident ischaemic stroke & No & \\
\hline \multirow[t]{2}{*}{ Ikram et $\left.a\right|^{26}$} & ROTT & 6780 & Inc ven calibre & Incident ischaemic stroke & Yes & 411 \\
\hline & & & Dec art calibre & Incident ischaemic stroke & Yes & \\
\hline \multirow[t]{2}{*}{ Wong et al ${ }^{18}$} & $\mathrm{CHS}$ & 1992 & Inc ven calibre & Incident stroke & Yes & 113 \\
\hline & & & Dec art calibre & Incident stroke & No & \\
\hline \multicolumn{2}{|l|}{ Howard and Russell ${ }^{42}$} & 85 & RAO & Incident stroke & Yes & 22 \\
\hline Klein et $a l^{20}$ & WESDR & 996 & Retinopathy & Incident stroke & Yes & 59 \\
\hline Cohen et $a^{43}$ & & 950 & Retinopathy & Incident stroke & Yes & 41 \\
\hline Kim et $a^{39}$ & & 365 & Retinopathy & Incident stroke & No & $?$ \\
\hline Fuller et $a^{44}$ & & 4753 & Retinopathy & Incident stroke & Yes & 293 \\
\hline Wong et $a l^{15}$ & ARIC & 1684 & Retinopathy & Incident stroke & Yes & 32 \\
\hline \multirow{4}{*}{ Mitchell et al ${ }^{11}$} & BMES & 3583 & Retinopathy & Incident stroke/TIA & Yes & 132 \\
\hline & & & FAN & Incident stroke/TIA & Yes & \\
\hline & & & AVN & Incident stroke/TIA & No & \\
\hline & & & AVR & Incident stroke/TIA & No & \\
\hline \multirow[t]{2}{*}{ Ikram et $a l^{27}$} & ROTT & 490 & Inc ven calibre & Incident MRI infarct & Yes & 33 \\
\hline & & & Dec art calibre & Incident MRI infarct & No & \\
\hline Klein et $a l^{22}$ & WESDR & 2366 & Retinopathy & Incident death with stroke & Yes & 274 \\
\hline Hirai et $a l^{19}$ & BDES & 4284 & Retinopathy & Incident death with stroke & No & $?$ \\
\hline Klein et $a l^{21}$ & BDES & 4856 & Retinal embolus & Incident death with stroke & Yes & 97 \\
\hline Wang et $a l^{12}$ & BMES/BDES & 8580 & Retinal embolus & Incident death with stroke & Yes & 344 \\
\hline \multirow[t]{3}{*}{ Witt et $a l^{13}$} & BDES & 684 & Dec art calibre & Incident death with stroke & No & 28 \\
\hline & & & Tortuosity & Incident death with stroke & No & \\
\hline & & & Bifurcation angle & Incident death with stroke & No & \\
\hline Klein et $a l^{24}$ & BDES & 4926 & Retinal emboli & Incident death with stroke & No & 154 \\
\hline Cugati et al ${ }^{28}$ & BDES/BMES & 8580 & RVO & Incident death with stroke & No & 341 \\
\hline Tsaloumas et $a l^{41}$ & & 95 & RVO & Incident death with stroke & No & 18 \\
\hline
\end{tabular}


12 papers used clinical assessment but not necessarily at the time of the stroke by study investigators; ; $^{11} 1720232532333539434414$ papers used phone interviews, review of case notes or death certificates; ${ }^{812-15} 181921222426284142$ and nine papers used brain imaging without clinical correlation. $^{9} 1027$ 29-31 36-38

\section{Ascertainment of retinal microvascular abnormalities}

The retinal assessment techniques were well described and performed. Twenty-seven of 37 performed retinal photography; ;-2934373840 43 six performed direct ophthalmoscopy; ${ }^{31} 3235414244$ two used hospital records; ${ }^{33} 39$ and only two did not record the method of retinal assessment. ${ }^{30}{ }^{36}$ Twentytwo of 37 papers used retinal abnormality classifications that had been validated either internally or externally. ${ }^{8-29}$ Of the 27 papers which used retinal photography, the number of fields used and the angle of the field of view varied: four papers gave no information regarding which retinal fields were taken, ${ }^{29} 34374015$ took one field centred on the optic disc and macula, ${ }^{9} 1013-16181921232426-2838$ and eight took more than one field. ${ }^{811} 121720222543$ Eight of 27 papers gave no information regarding the angle of the field of view used, , 20222934374043 two papers used 20 , ${ }^{26} 27$ eight papers 30 , ,1-13 1921242528 eight papers 45 $5^{\circ-10} 14-161823$ and one paper $50^{\circ} .38$

The retinal features that were assessed varied greatly, the most common being retinopathy (19/37 papers) defined as any of the following: microaneurysms, haemorrhages or exudates. ${ }^{8-}$ 11 14-17 192022 29-33 394344 Two papers divided retinopathy into its constituent parts and presented separately. ${ }^{9} 10$ Other papers used retinal artery occlusion/emboli (6/37 papers); ;2 $^{21} 24364042$ retinal vein occlusion (5/37 papers); ${ }^{25} 28353641$ vessel calibre of either the venules or arterioles, or generalised or focal narrowing (9/37 papers); ;-11 131418232627 arteriovenular nicking(5/37 papers); $;^{9-11} 1423$ retinal geometry (2/37 papers) $;^{13}{ }^{38}$ or retinal artery sclerosis (3/37 papers)..$^{34} 3788$

\section{Association between retinal microvascular abnormalities and stroke}

We were able to extract data and combine risk ratios for 24 papers (total study population 39 376, 1696 stroke (figs 1, 2).

\section{Retinopathy and stroke}

Retinopathy was associated with incident stroke; among eight papers providing data (total population 25 354; 1019 strokes), 811141519202244 the sRR for incident stroke in the presence versus absence of retinopathy was 2.1 (95\% CI 1.7 to 2.6; fig. 1) with no significant heterogeneity between papers $\left(\chi^{2}\right.$ $\mathrm{p}=0.46)$.

Retinopathy was associated with prevalent stroke; among seven papers providing data (total population 8083; 979 strokes),, 101617293233 the sRR for prevalent stroke in the presence versus absence of retinopathy was 2.45 (95\% CI 1.4 to 4.3; fig. 2) with significant heterogeneity between papers $\left(\chi^{2}\right.$ $\mathrm{p}=0.003)$

\section{Arteriolar and venular widths and stroke}

Decreased arteriolar width was not associated with incident stroke; among three papers providing data (total population 9262; 557 strokes), ${ }^{18} 2627$ decreasing arteriolar width was not associated with incident stroke-sRR 0.9 (95\% CI 0.8 to 1.1; fig. 1) with no significant heterogeneity between papers $\left(\chi^{2}\right.$ $\mathrm{p}=0.67$ )
Increased venular width was associated with incident stroke; among the same three papers providing data for this analysis (total population 9262; 557 strokes), ${ }^{18} 26{ }^{27}$ venular widening was associated with incident stroke-sRR 1.4 (95\% CI 1.1 to 1.7; fig. 1) with no significant heterogeneity between papers $\left(\chi^{2}\right.$ $\mathrm{p}=0.36)$. No papers assessed arteriolar or venular widths and prevalent stroke.

Six papers assessed the relationship between AVR and incident stroke, and we were able to extract data from five papers (21 717 subjects, 748 strokes). ${ }^{11} 14202627$ Decreased AVR was not associated with incident stroke (sRR 1.4, 95\% CI 0.9 to 2.0; fig 1), although there was significant heterogeneity between these papers $\left(\chi^{2} p=0.002\right)$. Three further papers found a significant association between decreased AVR and prevalent stroke (5451 subjects, 812 strokes) ${ }^{9} 1016$ with sRR 1.2 (95\% CI 1.1 to 1.3 ; fig. 2) with no significant heterogeneity between papers $\left(\chi^{2} p=0.47\right)$, but another paper from which we could not extract data reported no association between AVR and prevalent stroke. ${ }^{23}$

\section{Retinal arteriolar emboli or arteriolar occlusion and stroke}

Six papers assessed retinal emboli and incident stroke $e^{12} 2124364042$ from which we could extract data from four papers $^{12} 212440$ but Klein et al present 5-year follow-up data from the BDES, and Klein et $a^{24}$ present a 10 -year follow-up from the same patients; thus when combining studies, we excluded Klein et al. ${ }^{21}$ Furthermore, Wang et a ${ }^{12}$ presented a pooled analysis of data from the BDES and the BMES. As the data from the BDES are included in Klein et al, ${ }^{24}$ we present in this analysis the data presented in Wang et a ${ }^{12}$ from the BMES (to avoid double counting), so a total of 8720 subjects and 266 strokes gave an sRR of 2.9 (95\% CI 1.6 to 5.1; fig. 1) with no significant heterogeneity between papers $\left(\chi^{2} p=0.21\right)$. One paper assessed retinal artery occlusion and prevalent stroke, but we were unable to extract any data. ${ }^{36}$

\section{Retinal vein occlusion and stroke}

Two papers that investigated the association between RVO and incident stroke (9168 subjects; 359 strokes) found no association-sRR 1.2 (95\% CI 0.8 to 1.9; fig. 1$)^{28}{ }^{41}$ with no significant heterogeneity between papers $\left(\chi^{2} p=0.37\right)$. A further two papers studied RVO and prevalent stroke (4744 subjects, 43 strokes) - the one paper (3654 subjects) from which we could extract data found RVO was associated with prevalent strokes (sRR 3.8, 95\% CI 1.9 to 7.6; fig. 2). ${ }^{25}$

\section{Retinal microvascular abnormalities in ischaemic versus haemorrhagic stroke}

No papers directly compared and reported differences between retinal microvascular abnormalities in ischaemic versus haemorrhagic stroke.

\section{Retinal microvascular abnormalities and large artery versus small artery stroke}

Only one pilot study ${ }^{34}$ directly compared retinal microvascular abnormalities in ischaemic stroke subtypes. The diagnosis of stroke and subtyping was based on clinical and radiological features at the time of the stroke. This small study (59 patients in total) found that there were similarly high levels of mild retinal arteriosclerosis (defined as altered central arteriolar light reflex) in lacunar stroke (prevalence 92\%) and cortical stroke (prevalence $80 \%$ ). 
Retinopathy

Risk ratio

(95\% Cl)

$2.6(1.6-4.3)$

Wong ${ }^{14} 2001$ (ARIC)

Wong ${ }^{15} 2002$ (ARIC)

Mitchel|11 2005 (BMES)

Klein22 1999 (WESDR)

Klein 202004 (WESDR)

Hirai19 2007 (BDES)

Fuller44 2001

Cheung ${ }^{8} 2007$ (ARIC)

sRR retinopathy

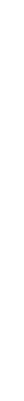

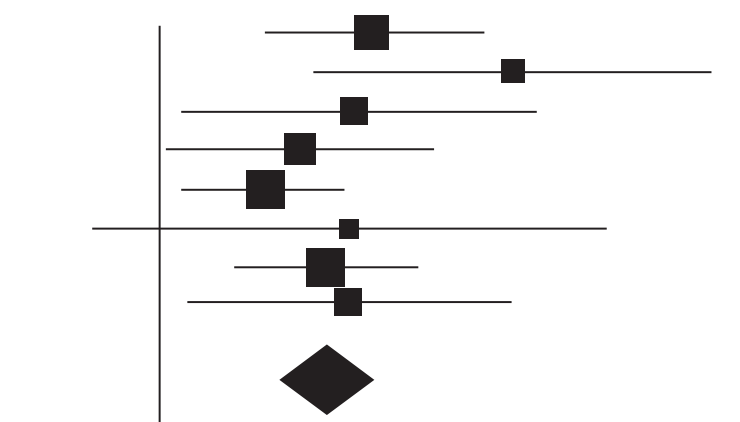

$4.9(2.0-11.9)$

$2.4(1.1-5.4)$

$1.9(1.0-3.4)$

$1.6(1.1-2.3)$

$2.3(0.7-7.4)$

$2.1(1.4-3.2)$

$2.3(1.1-4.9)$

$2.1(1.7-2.6)$

Arteriolar narrowing

$$
\begin{aligned}
& \text { Wong }{ }^{18} 2006 \text { (CHS) } \\
& \text { Ikram²6 } 2006 \text { (ROTT) }^{20} \text { lkram²7 2006* (ROTT) }^{200} \text { (ROT) }
\end{aligned}
$$

sRR arteriolar narrowing

Venular widening

$$
\begin{aligned}
& \text { Wong }^{18} 2006 \text { (CHS) } \\
& \text { lkram }^{26} 2006 \text { (ROTT) } \\
& \text { lkram }^{27} 2006^{*} \text { (ROTT) } \\
& \text { sRR venular widening }
\end{aligned}
$$

Decreased AVR

Klein20 2004 (WESDR)

Wong ${ }^{14} 2001$ (ARIC)

Mitchell11 2005 (BMES)

Ikram $^{26} 2006$ (ROTT)

Ikram $^{27}$ 2006* (ROTT)

sRR decreased AVR

Retinal artery occlusion

$$
\begin{aligned}
& \text { Wang }^{12} 2006 \text { (BMES) } \\
& \text { Klein }^{24} 2003 \text { (BDES) } \\
& \text { Bruno40 } 1995
\end{aligned}
$$

sRR retinal artery occlusion

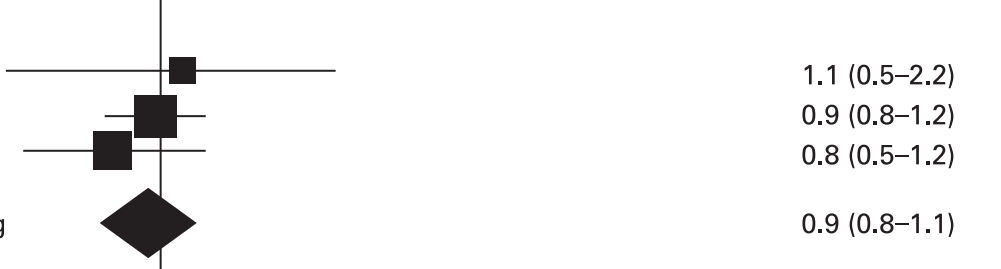

Age sex BP DM

Age sex

Age sex BP DM

$2.2(1.1-4.3)$

$1.3(1.0-1.6)$

$1.2(0.7-2.2)$

$1.4(1.1-1.7)$

$0.8(0.6-1.1)$

$2.3(1.2-4.3)$

$1.7(0.8-4.0)$

$1.6(1.3-2.1)$

$1.3(0.8-2.0)$

$1.4(0.9-2.0)$

$2.1(0.7-6.9)$

$2.4(1.1-5.0)$

$9.9(2.3-43.1)$

$2.9(1.6-5.1)$

Retinal vein occlusion

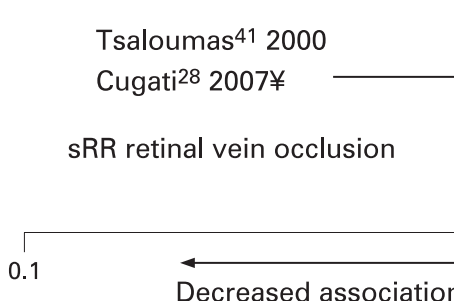

Tsaloumas ${ }^{41} 2000$

$\overleftarrow{\text { Decreased association }}$

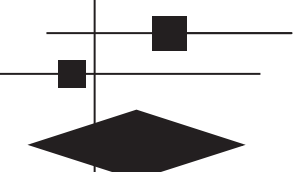

$1.4(0.8-2.4)$

Nil

$0.9(0.4-2.1)$

Age sex BP DM

$1.2(0.8-1.9)$

Log risk ratio

Adjusted for

Age sex BP DM

Age sex BP DM

Age sex BP DM

Age sex BP DM

DM

Age sex BP DM

DM

Age sex BP DM

Age sex BP DM

Age sex

Age sex BP DM

DM

Age sex BP DM

Age sex BP DM

Age sex

Age sex BP DM

Age sex BP

Age sex BP

Age sex

Figure 1 Summary risk ratios (sRR) showing association of incident stroke and different retinal microvascular abnormalities. The size of the square denotes the weight attributed to each paper, and the horizontal lines represent the $95 \% \mathrm{Cl}$. A diamond represents sRR with the width representing the $95 \% \mathrm{Cl}$. An arrowhead mean that the upper $\mathrm{Cl}$ is not marked on chart. The right-hand column indicates key adjustments. BP, blood pressure; DM, diabetes mellitus. ${ }^{*}$ Studies which used only radiological diagnosis of infarct; $¥ B M E S / B D E S$ combined. 
Table 2 Background information of population-based studies contributing to the review

\begin{tabular}{|c|c|c|c|c|}
\hline Study & Acronym & Size & Age (years) & Description \\
\hline Atherosclerosis Risk in Communities Study & ARIC & 15792 & $45-64$ & $\begin{array}{l}\text { Longitudinal US population-based study of cardiovascular } \\
\text { disease }\end{array}$ \\
\hline Beaver Dam Eye Study & BDES & 4926 & $43-84$ & $\begin{array}{l}\text { Longitudinal US population-based study of eye disease and } \\
\text { medical health }\end{array}$ \\
\hline Cardiovascular Health Study & CHS & 5201 & $>65$ & $\begin{array}{l}\text { Longitudinal US population-based study of coronary heart } \\
\text { disease and stroke }\end{array}$ \\
\hline Rotterdam Study & ROTT & 7983 & $>55$ & $\begin{array}{l}\text { Longitudinal Dutch population-based study of chronic } \\
\text { diseases }\end{array}$ \\
\hline Blue Mountains Eye Study & BMES & 3654 & $>49$ & $\begin{array}{l}\text { Longitudinal Australian population-based study of vision, } \\
\text { common eye diseases and health outcomes }\end{array}$ \\
\hline $\begin{array}{l}\text { Wisconsin Epidemiological Study of Diabetic } \\
\text { Retinopathy }\end{array}$ & WESDR & 996 & All & $\begin{array}{l}\text { Longitudinal US study of patients receiving treatment for } \\
\text { type I diabetes }\end{array}$ \\
\hline Australian Diabetes, Obesity and Lifestyle Study & Ausdiab & 11247 & $>25$ & Cross-sectional Australian population-based survey \\
\hline
\end{tabular}

\section{Retinal microvascular abnormalities and transient ischaemic attacks}

No papers solely examined retinal disease and TIA. Two papers that included TIAs in their stroke outcomes did not present separate data for TIA. ${ }^{11} 32$

\section{DISCUSSION}

This review has demonstrated an association between retinal microvascular abnormalities and any stroke despite large variations in study design, stroke outcomes used and a lack of detail in diagnosing both the presence and type of stroke. It has shown that the presence of retinopathy is associated with incident stroke and prevalent stroke. Furthermore, incident stroke was associated with retinal artery embolism, venular widening and decreased AVR but not with arteriolar narrowing.
There were no data on arteriolar narrowing or venular widening and prevalent stroke, and decreased AVR was not associated with prevalent stroke. Thus, there were some inconsistencies in results, which, combined with the heterogeneity between studies for some analyses, means that the data on different types of retinal microvascular abnormality and stroke should be viewed cautiously. No published studies have adequately compared retinal microvascular abnormalities in haemorrhagic versus ischaemic stroke or between different ischaemic stroke subtypes, so we are unable to answer one of our initial hypotheses that retinal microvascular abnormalities may shed light on the pathophysiology of small vessel disease.

Population-based studies contributed the majority of papers, and so the total number of subjects was large (62 975 subjects), but in healthy and relatively young populations (mean age

\begin{tabular}{|c|c|}
\hline \multicolumn{2}{|l|}{ Retinopathy } \\
\hline & Wong ${ }^{16} 2003$ (CHS) \\
\hline & Wong17 2005 \\
\hline & Petitti33 1995 \\
\hline & Longstreth ${ }^{10} 2006^{*}$ (CHS) \\
\hline & Kwon $292006^{*}$ \\
\hline & Cooper ${ }^{9} 2005^{*}(A R I C)$ \\
\hline & Abu El Asrar 322002 \\
\hline & sRR retinopathy \\
\hline
\end{tabular}

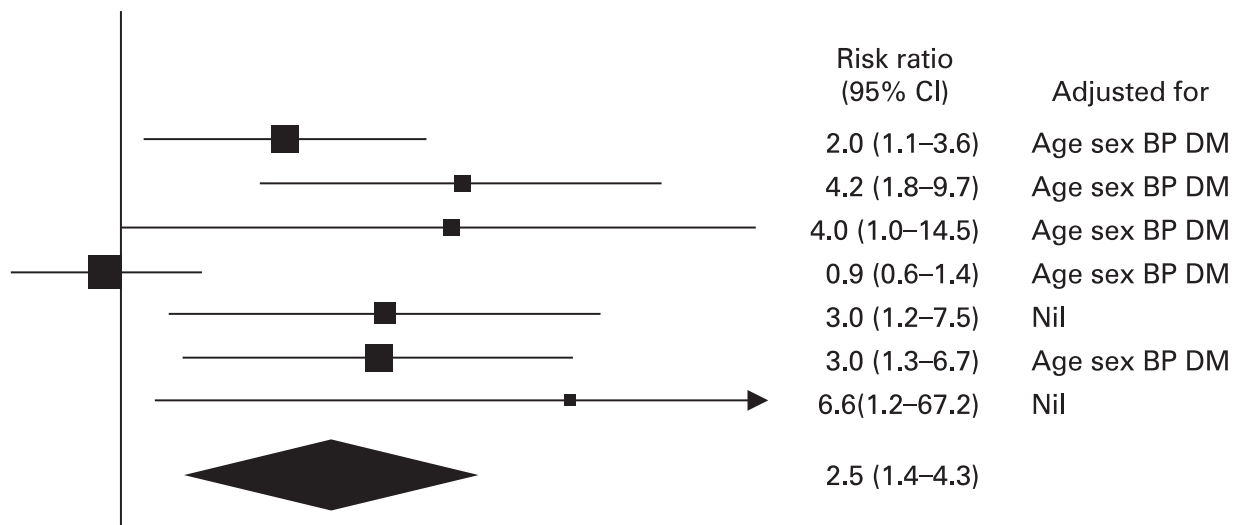

Decreased AVR

Wong ${ }^{16} 2003$ (CHS)
Longstreth ${ }^{10} 2006^{*}$ (CHS)
Cooper ${ }^{2005^{*}}$ (ARIC)
sRR decreased AVR

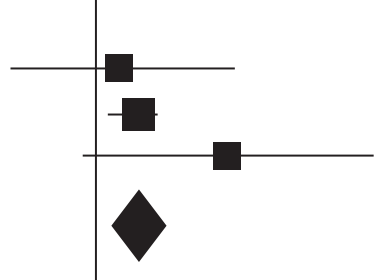

$1.1(0.7-1.8)$

Age sex BP DM

$1.2(1.1-1.3) \quad$ Age sex BP DM

$1.7(0.9-3.2) \quad$ Age sex BP DM

$1.2(1.1-1.3)$

Retinal vein occlusion

\begin{tabular}{|c|c|c|}
\hline & Mitchell25 1996 (BMES) & \\
\hline 0.1 & $\longleftarrow$ Decreased association & 100 \\
\hline
\end{tabular}

Figure 2 Summary risk ratios (sRR) showing association of prevalent stroke and different retinal microvascular abnormalities. The size of the square denotes the weight attributed to each paper, and the horizontal lines represent the $95 \% \mathrm{Cl}$. Diamonds represent the sRR, and the width represents the $95 \% \mathrm{Cl}$. Risk ratios for Abu El-Asrar ${ }^{32}$ were calculated directly from raw data. An arrowhead means that the upper $\mathrm{Cl}$ is not marked on the chart. The right-hand column indicates key adjustments. BP, blood pressure; DM, diabetes mellitus. ${ }^{*}$ Studies which used only radiological diagnosis of infarct. 
62.5 years, younger than the mean age of stroke onset in the UK of 72 years) stroke is rare, making it difficult to show differences between exposure groups. The rareness of strokes is reflected in wide $95 \% \mathrm{CI},{ }^{15}$ the heterogeneity between studies for some associations and the occasional need to combine cohorts to demonstrate associations in some primary publications, ${ }^{12}$ indicating that the data on specific retinal features and stroke should be viewed with caution. Although the general association between any retinal microvascular abnormalities and stroke may be robust, the details of the associations between different retinal microvascular features and stroke are inconsistent and may be less reliable. Case control studies can be used when the outcome of interest is rare, but studies in this review used the general population as controls ${ }^{354142}$ or MRI scans of patients who self-presented to hospital for general health checks. ${ }^{37}$ We were unable to perform any subanalysis by age group, but it is likely that older populations and populations where all patients have had a stroke will have more retinal abnormalities, thereby increasing the chance of finding associations between retinal changes and stroke subtypes.

The overall results of the review are promising in that for most analyses, the heterogeneity between studies is low. However, there are inconsistencies. For example, it is odd that decreased AVR should be associated with prevalent stroke but not incident stroke which could be due to the significant heterogeneity in the results from the incident stroke papers (perhaps resulting from Klein et a ${ }^{20}$ using patients with type I diabetes as their cohort who were younger than the patients in the other studies) possibly causing a type II statistical error for the association between decreased AVR and incident stroke or recall bias affecting the detection of prevalent stroke. Although there were a similar number of strokes, there were many more subjects in the analysis of incident stroke than of prevalent stroke and AVR. Similarly there is significant heterogeneity between the studies for retinopathy and prevalent stroke but not retinopathy and incident stroke, this may reflect the wide variety of methods used to identify and diagnose prevalent stroke as the number of strokes in the two analyses were similar. Some heterogeneity in the association between retinopathy and prevalent stroke may have resulted from Longstreth et $a l^{10}$ investigating associations with MRI defined infarcts in the Cardiovascular Health Study which involved an older age group ( $>65$ years) than the others in the subgroup investigating retinopathy and prevalent stroke. Although we have not demonstrated much heterogeneity, it should be noted that with small numbers of outcomes, the power to detect heterogeneity is low, and our calculations may have missed a small degree of heterogeneity.

Stroke is difficult to diagnose. Ideally, the patient should be assessed by a suitably trained stroke expert at the time of the stroke and the diagnosis made with clinical features and appropriately timed brain imaging. Many of the incident stroke studies were population-based, and it is almost impossible for study investigators to assess every possible stroke. Accordingly, the diagnosis of stroke was made at either regular review when patients were asked if they had had a stroke in the preceding follow-up period and medical records reviewed, or patients were asked to report possible strokes to the investigators. Alternatively, discharge summaries or even death certificates were used to diagnose stroke. Although these techniques allow for the assessment of large numbers of subjects, they come at the price of reduced accuracy, ${ }^{45-47}$ as discharge summaries and death certificates are often completed by inexperienced junior medical staff. To ascertain whether a patient had a history of stroke, many studies asked the patient and searched medical records. Although prone to recall bias, this approach is deemed acceptable for assessing prevalent stroke epidemiologically, but may be inadequate for detailed studies of pathophysiology. We have grouped together studies investigating certain retinal features and stroke outcomes but there were varying methods of both retinal assessment and more pertinently stroke assessment. We have tried to minimise this variation but it is a limitation of the results of this review that this variation exists.

In order to assess for a small study bias, we plotted a Funnel plot of the logarithm of the effect size and standard error of the effect size, and found that there was a paucity of small negative studies. Furthermore, the larger studies tended to have smaller effect sizes detailing the association between retinal microvascular abnormalities and stroke. One of the possible causes of this small study bias is publication bias, and it is likely that publication bias has affected the results of this review.

Stroke is a heterogeneous disorder. The two main pathophysiological divisions are haemorrhagic and ischaemic stroke, and ischaemic stroke is further subtyped into lacunar (or small vessel disease) and cortical (large artery atheroma and cardiac embolism subtypes). Furthermore, while stroke subtypes share many vascular risk factors, their underlying pathophysiology varies, reflecting different disease processes. Any study aiming to understand the pathophysiology of stroke, or even to assess novel risk factors for stroke, should subdivide stroke into ischaemic and haemorrhagic, and preferably further subtype ischaemic stroke. This requires careful clinical assessment and appropriate brain imaging. In the ARIC study, strokes were subtyped according to their likely cause based on clinical and imaging criteria where available-haemorrhagic or ischaemic with ischaemic strokes further subtyped into the important subdivisions of thrombotic or embolic stroke but not lacunar versus cortical. However, due to the small number of strokes (and especially haemorrhagic strokes) ${ }^{14} 15$ direct comparisons between stroke subtypes were not reported. The Rotterdam Study investigated cerebral infarction (but brain imaging was only available for $68 \%$ of patients). ${ }^{26}$ Other papers subtyped stroke but did not compare retinal microvascular abnormalities between the different stroke subtypes but only between any stroke and non-stroke controls. ${ }^{33} 40$ One study that did compare retinal microvascular abnormalities in lacunar and cortical stroke directly and had adequate diagnosis and subtyping ${ }^{34}$ was underpowered $(n=60)$ and used subjective measures of retinal arteriolar sclerosis (altered central light reflex).

MRI used appropriately is a powerful tool for diagnosing and subtyping stroke, but in the absence of clinical data about neurological deficits, the abnormal areas seen on imaging are difficult to interpret, especially as they are common in older populations. ${ }^{48}$ Lacunar infarcts can have considerable overlap with white-matter lesions. We excluded studies dealing solely with white-matter lesions, but some of the included studies may have misclassified white-matter lesions as lacunes and vice versa, thus introducing a small bias. Without detailed diffusion weighted MR imaging, or careful computed tomography assessment at the time of any symptoms, it is not clear which, if any, lacunes may have represented previous clinically evident stroke. As such, stroke remains a clinical diagnosis, and associations between retinal features and lacunes seen on MRI may be less reliable in aiding to understand the pathophysiology of stroke subtypes.

A few earlier studies used direct ophthalmoscopy to record retinal microvascular abnormalities. Apart from this, the quality 
of retinal assessment used in most papers was high. Almost all of the later studies, and certainly all of the large populationbased cohort studies, used retinal photography with grading by trained analysts using internally and externally validated techniques blinded to other clinical information.

The strength of the associations between retinal microvascular abnormalities and stroke is encouraging, especially given the consistency between studies. This suggests that retinal examination offers an excellent way to study non-invasively the effects of common vascular risk factors on small vessels and possibly for gaining a better understanding of the pathophysiological processes involved in cerebral small vessel disease. The lack of studies which directly compare retinal microvascular abnormalities and stroke ischaemic subtypes is therefore disappointing, and further studies carefully characterising stroke subtype with comprehensive assessment at the time of the stroke are required if retinal microvascular abnormalities are to shed light on the pathophysiology of lacunar stroke. The paucity of strokes, lack of detailed stroke assessment and heterogeneity between studies for some associations mean that the data on individual retinal features, and stroke should be viewed with caution and underpins the need for more large, stroke-focussed, robust studies.

Funding: FND is supported by the Wellcome Trust (grant number 075611), who had no role in the conception or writing of this review.

Competing interests: None.

\section{REFERENCES}

1. Sudlow CLM, Warlow CP. Comparable studies of the incidence of stroke and its pathological types. Results from an international collaboration. Stroke 1997;28:491-9.

2. Wardlaw JM. What causes lacunar stroke? J Neurol Neurosurg Psychiatry 2005;76:617-19.

3. Macdonald RL, Kowalczuk A, Johns L. Emboli enter penetrating arteries of monkey brain in relation to their size. Stroke 1995;26:1247-51.

4. Wardlaw JM, Farrall A, Armitage PA, et al. Changes in background blood-brain barrier integrity between lacunar and cortical ischemic stroke subtypes. Stroke 2008;39:1327-32.

5. Patton N, Aslam T, Macgillivray T, et al. Retinal vascular image analysis as a potential screening tool for cerebrovascular disease: a rationale based on homology between cerebral and retinal microvasculatures. J Anat 2005;206:319-48.

6. Wong TY, Mclntosh R. Systemic associations of retinal microvascular signs: a review of recent population-based studies. Ophthalmic Physiol Opt 2005;25:195-204.

7. Hatano S. Experience from a multicentre stroke register: a preliminary report. Bull World Health Org 1976. Report No. 54:541-53.

8. Cheung $\mathbf{N}$, Rogers $\mathbf{S}$, Couper DJ, et al. Is diabetic retinopathy an independent risk factor for ischemic stroke? Stroke 2007:38:398-401.

9. Cooper LS, Wong TY, Klein R, et al. Retinal microvascular abnormalities and MRIdefined subclinical cerebral infarction: the Atherosclerosis Risk in Communities Study. Stroke 2006:37:82-6.

10. Longstreth W Jr, Larsen EK, Klein R, et al. Associations between findings on cranial magnetic resonance imaging and retinal photography in the elderly: the Cardiovascular Health Study. Am J Epidemiol 2007;165:78-84.

11. Mitchell P, Wang JJ, Wong TY, et al. Retinal microvascular signs and risk of stroke and stroke mortality. Neurology 2005;65:1005-9.

12. Wang JJ, Cugati S, Knudtson MD, et al. Retinal arteriolar emboli and long-term mortality: pooled data analysis from two older populations. Stroke 2006;37:1833-6.

13. Witt N, Wong TY, Hughes AD, et al. Abnormalities of retinal microvascular structure and risk of mortality from ischemic heart disease and stroke. Hypertension 2006;47:975-81.

14. Wong TY, Klein R, Couper DJ, et al. Retinal microvascular abnormalities and incident stroke: The Atherosclerosis Risk in Communities Study. Lancet 2001;358:1134-40.

15. Wong TY, Klein R, Sharrett AR, et al. Cerebral white matter lesions, retinopathy, and incident clinical stroke. J Am Med Assoc 2002:288:67-74.

16. Wong TY, Klein R, Sharrett AR, et al. The prevalence and risk factors of retinal microvascular abnormalities in older persons: The Cardiovascular Health Study. Ophthalmology 2003;110:658-66.
17. Wong TY, Barr ELM, Tapp RJ, et al. Retinopathy in persons with impaired glucose metabolism: The Australian Diabetes Obesity and Lifestyle (AusDiab) study. Am J Ophthalmol 2005;140:1157-9.

18. Wong TY, Kamineni A, Klein R, et al. Quantitative retinal venular caliber and risk of cardiovascular disease in older persons: the cardiovascular health study. Arch Intern Med 2006;166:2388-94.

19. Hirai FE, Moss SE, Knudtson MD, et al. Retinopathy and survival in a population without diabetes: the Beaver Dam Eye Study. Am J Epidemiol 2007;166:724-30.

20. Klein BEK, Klein R, McBride PE, et al. Cardiovascular disease, mortality, and retinal microvascular characteristics in type 1 diabetes: Wisconsin Epidemiologic Study of Diabetic Retinopathy. Arch Intern Med 2004:164:1917-24.

21. Klein R, Klein BEK, Jensen SC, et al. Retinal emboli and stroke: The Beaver Dam Eye Study. Arch Ophthalmol 1999;117:1063-8.

22. Klein R, Klein BEK, Moss SE, et al. Association of ocular disease and mortality in a diabetic population. Arch Ophthalmol 1999:117:1487-95.

23. Klein R, Sharrett AR, Klein BEK, et al. Are retinal arteriolar abnormalities related to atherosclerosis? The Atherosclerosis Risk in Communities Study. Arterioscler Thromb Vasc Biol 2000;20:1644-50.

24. Klein R, Klein BEK, Moss SE, et al. Retinal emboli and cardiovascular disease: The Beaver Dam Eye Study. Trans Am Ophthalmol Soc 2003;101:173-82.

25. Mitchell P, Smith W, Chang A. Prevalence and associations of retinal vein occlusion in Australia: the Blue Mountains Eye Study. Arch Ophthalmol 1996;114:1243-7.

26. Ikram MK, De Jong FJ, Bos MJ, et al. Retinal vessel diameters and risk of stroke: the Rotterdam Study. Neurology 2006;66:1339-43.

27. Ikram MK, De Jong FJ, Van Dijk EJ, et al. Retinal vessel diameters and cerebral small vessel disease: the Rotterdam Scan Study. Brain 2006:129:182-8.

28. Cugati S, Wang JJ, Knudtson MD, et al. Retinal vein occlusion and vascular mortality. Pooled data analysis of two population-based cohorts. Ophthalmology 2007:114:520-4.

29. Kwon H-M, Kim BJ, Oh JY, et al. Retinopathy as an indicator of silent brain infarction in asymptomatic hypertensive subjects. J Neurol Sci 2007:252:159-62.

30. Inoue T, Fushimi H, Yamada Y, et al. Asymptomatic multiple lacunae in diabetics and non-diabetics detected by brain magnetic resonance imaging. Diabetes Res Clin Pract 1996;31:81-6.

31. Ikeda T, Gomi T, Kobayashi S, et al. Role of hypertension in asymptomatic cerebral lacunae in the elderly. Hypertension 1994;23(Suppl):I259-62S

32. Abu El-Asrar AM, Al Rubeaan KA, Al Amro SA, et al. Retinopathy as a predictor of other diabetic complications. Int Ophthalmol 2001;24:1-11.

33. Petitti DB, Bhatt $H$. Retinopathy as a risk factor for nonembolic stroke in diabetic subjects. Stroke 1995;26:593-6.

34. Luijckx GJ, Boiten J, Kroonenburgh Mv, et al. Systemic small-vessel disease is not exclusively related to lacunar stroke. A pilot study. J Stroke Cerebrovasc Dis 1998; 7:52-7

35. Hayreh SS, Zimmerman B, McCarthy MJ, et al. Systemic diseases associated with various types of retinal vein occlusion. Am J Ophthalmol 2001:131:61-77.

36. Ueda Y, Kanazawa $S$, Ohira A, et al. Retinal vascular obstruction and asymptomatic cerebral infarction. Jpn J Ophthalmol 2002:46:209-14.

37. Kobayashi S, Okada K, Koide H, et al. Subcortical silent brain infarction as a risk factor for clinical stroke. Stroke 1997;28:1932-9

38. Kwa VIH, Van der Sande JJ, Stam J, et al. Retinal arterial changes correlate with cerebral small-vessel disease. Neurology 2002;59:1536-40.

39. Kim Y-H, Hong M-K, Lee CW, et al. Diabetic retinopathy as a predictor of late clinical events following percutaneous coronary intervention. J Invasive Cardiol 2002; 14:599-602

40. Bruno A, Jones WL, Austin JK, et al. Vascular outcome in men with asymptomatic retinal cholesterol emboli. A cohort study. Ann Intern Med 1995:122:249-53.

41. Tsaloumas MD, Kirwan J, Vinall H, et al. Nine year follow-up study of morbidity and mortality in retinal vein occlusion. Eve 2000;14:821-7.

42. Howard RS, Russell RWR. Prognosis of patients with retinal embolism. J Neurol Neurosurg Psychiatry 1987; 50:1142-7.

43. Cohen JA, Estacio R0, Lundgren RA, et al. Diabetic autonomic neuropathy is associated with an increased incidence of strokes. Auton Neurosci 2003;108:73-8.

44. Fuller JH, Stevens LK, Wang S-L, et al. Risk factors for cardiovascular mortality and morbidity: The WHO multinational study of vascular disease in diabetes. Diabetologia 2001;44(2 Suppl):54-64S

45. Leibson CL, Naessens JM, Brown RD, et al. Accuracy of hospital discharge abstracts for identifying stroke. Stroke 1994;25:2348-55.

46. Leppala JM, Virtamo J, Heinonen OP. Validation of stroke diagnosis in the national hospital discharge register and the register of causes of death in Finland. Eur J Epidemiol 1999;15:155-60.

47. Piriyawat $\mathbf{P}$, Smajsova M, Smith MA, et al. Comparison of active and passive surveillance for cerebrovascular disease: the brain attack surveillance in Corpus Christi (BASIC) Project. Am J Epidemiol 2002;156:1062-9.

48. Vernooij MW, Ikram MA, Tanghe $\mathrm{HL}$, et al. Incidental findings on brain MRI in the general population. N Engl J Med 2007;357:1821-8. 